\title{
Perfil Fisiológico y Rendimiento Deportivo en Jugadoras de Baloncesto Universitario
}

\author{
Omar Jesús Salido Angulo, M.C \\ Omar Ricardo Ortiz Gómez, M.C \\ Dr. Karina Denisse Moguel Torres, \\ Ramón Lugo Parra, M.C \\ Ricardo Gumaro Molina Jacquez, M.C \\ Samuel Alfredo Islas Guerra, M.P.A \\ Arturo Iván Chávez Erives, M.A.P.E \\ Facultad de Ciencias de la Cultura, \\ Universidad Autónoma de Chihuahua, México
}

Doi:10.19044/esj.2020.v16n26p11 URL:http://dx.doi.org/10.19044/esj.2020.v16n26p11

\section{Resumen}

Propósito: identificar diferencias en capacidades físicas y tiro libre en jugadoras de baloncesto universitario por posición de juego. Método: quince jugadoras de baloncesto representativas de la Universidad Autónoma de Chihuahua, edad $21.7( \pm 2.5)$ años, peso $70.7(8.6) \mathrm{kg}$, altura $170(7.5) \mathrm{cm}$ y la experiencia de baloncesto de 10.0 (3.6) años se dividieron de acuerdo con su posición de juego en grupos de Point Guard (PG) $(\mathrm{n}=6)$, Forward (PF) (n $=5)$ y Center $(C)(n=4)$. El perfil físico específico del baloncesto incluyó la capacidad aeróbica estimada con la Prueba de recuperación intermitente YoYo, agilidad (prueba de agilidad de baloncesto), velocidad (prueba de velocidad de cancha 3/4), salto vertical (contramovimiento vertical prueba de salto), determinación de porcentaje de tiro libre estático y dinámico. No se detectaron diferencias significativas en las comparaciones entre grupos en ninguna de las variables, lo cual no es de esperarse debido a que se ha reportado que los jugadores PG tienden a ser más veloces, ágiles y con mayor potencia en piernas que los centros, evidenciando el bajo nivel de los PG, mismos que presentaron un IMC con sobrepeso. Conclusión: se encontraron valores homogéneos entre los grupos, lo que no es representativo de la literatura en la que PG y PF presentan mejor estado físico.

Palabras Clave: Capacidad física, baloncesto, jugadoras universitarias 


\title{
Physiological Profile and Sports Performance in College Basketball Players
}

\author{
Omar Jesús Salido Angulo, M.C \\ Omar Ricardo Ortiz Gómez, M.C \\ Dr. Karina Denisse Moguel Torres, \\ Ramón Lugo Parra, M.C \\ Ricardo Gumaro Molina Jacquez, M.C \\ Samuel Alfredo Islas Guerra, M.P.A \\ Arturo Iván Chávez Erives, M.A.P.E \\ Facultad de Ciencias de la Cultura, \\ Universidad Autónoma de Chihuahua, México
}

\begin{abstract}
Purpose: to identify differences in physical abilities and free throw shoot in college basketball players by playing position. Method: fifteen representative basketball players from the Autonomous University of Chihuahua, age 21.7 ( \pm 2.5 ) years, weight $70.7(8.6) \mathrm{kg}$, height $170(7.5) \mathrm{cm}$ and basketball experience of 10.0 (3.6) years were divided according with their playing position in groups of Point Guard $(\mathrm{PG})(\mathrm{n}=6)$, Forward $(\mathrm{PF})(\mathrm{n}=5)$ and Center $(C)(n=4)$. Basketball specific physical profile included aerobic capacity estimated with the YoYo Intermittent Recovery Test, agility (basketball agility test), speed (3/4 court speed test), vertical jump (vertical countermovement jump test), Determination of percentage of static and dynamic free kick. No significant differences were detected in the comparisons between groups in any of the variables, which is not to be expected because it has been reported that PG players tend to be faster, more agile and with more power in the legs than the centers, evidencing the low level of the GPs, who presented an overweight BMI. Conclusion: homogeneous values were found between the groups, which is not representative of the literature in which PG and PF present better physical condition.
\end{abstract}

Keywords: Physical ability, basketball, college sports players 


\section{Introduction}

El baloncesto es una disciplina deportiva de equipo que requiere la ejecución de esfuerzos físicos intermitente de alta intensidad por lo tanto exige una buena forma física aerobia y anaerobia (Metaxas, Koutlianos, Sendelides, \& Mandroukas, 2009). Dentro del esquema aerobio, se conoce el parámetro consumo máximo de oxígeno $\left(\mathrm{VO}_{2} \mathrm{máx}\right)$ cuya definición expresa la tasa más alta a la que se puede absorber y utilizar oxígeno, por el cuerpo, durante ejercicios intensos. Para valorar este parámetro, existen métodos de laboratorio que miden directamente este tributo, sin embargo, también se han desarrollado test de campo con amplia validez y confiabilidad (Koutlianos et al., 2013). Uno de las pruebas es el YoYo Intermitten Recovery Test que fue inicialmente validado para futbolistas (Krustrup et al., 2003), sin embargo también se ha probado en basquetbolistas (Castagna, Impellizzeri, Rampinini, D'Ottavio, \& Manzi, 2008) y otros deportes (Kilic-Toprak et al., 2015; Zambak, 2019).

Además de la capacidad aerobia, serán necesarias otras capacidades del tipo anaerobio como el salto, agilidad y velocidad factores que han sido determinantes en la preparación física de los basquetbolistas y específicamente por posición de juego ya que los requerimientos serán distintos para un guardia que para un centro (Delextrat \& Cohen, 2009) independientemente del nivel deportivo (Latin, Berg, \& Baechle, 1994).

Las acciones durante un juego de baloncesto incluyen una variedad de movimientos, como correr, driblar y saltar, pero, que finalmente culminan en acciones de tiro a la canasta, que es la única forma de conseguir puntos. Diversos estudios han señalado las diferencias en la técnica que son inherentes de una correcta ejecución, por ejemplo desde el punto de vista cinético se denotan diferencias en el ángulo y velocidad de salida de la pelota (Miller \& Bartlett, 1996) e inclusive se han reportado los ajustes en la técnica como consecuencia de un oponente (Rojas, Cepero, Ona, \& Gutierrez, 2000).

El tiro libre por otro lado, es diferente a la gama de posibilidades que ocurren en ese deporte, ya que se realiza sin presión del oponente y siempre en la misma dirección y distancia además de que se ha reportado mayor efectividad en equipos ganadores con respecto a equipos no ganadores (Csataljay, O’Donoghue, Hughes, \& Dancs, 2009). Por esta razón el objetivo de esta investigación es identificar diferencias en capacidades físicas y tiro libre en jugadoras de baloncesto universitario por posición de juego.

\section{Método}

Sujetos. La muestra seleccionada estuvo constituida por 15 mujeres jugadoras de baloncesto representativas del equipo selectivo de la Universidad Autónoma de Chihuahua, México de las cuáles se consideraron los siguientes datos biométricos: edad promedio $21.7( \pm 2.5)$ años, peso promedio $70.7(8.6)$ 
$\mathrm{kg}$, estatura promedio $170(7.5) \mathrm{cm}$ y experticia promedio en ese deporte de 10.0 (3.6) años. Además, para el estudio las participantes fueron divididas por posición de juego según la posición natural que desempeñan tanto en los entrenamientos como en los juegos de la siguiente manera: "point guard" (PG) $(n=6)$, "power forwards" (PF) $(n=5)$ y "centers" (C) $(n=4)$. Para su reclutamiento, se solicitó autorización al entrenador del equipo, luego se explicaron los procedimientos y la mecánica de evaluación. Los sujetos firmaron una carta de consentimiento informado en donde se declara su participación voluntaria, riegos y pormenores de la ejecución además de poderse retirar del estudio o solicitar información adicional si fuese necesario. Se consideró la declaración de Helsinki como norma rectora en el tratamiento con seres humanos.

Valoración del IMC

Se determinó el índice de masa corporal (IMC) utilizando la fórmula $\mathrm{IMC}=$ peso $(\mathrm{kg}) /$ estatura $(\mathrm{m} 2)$.

\section{Valoración de capacidades físicas}

Capacidad aerobia. Se estimó el consumo máximo de oxígeno ( $\mathrm{VO}_{2}$ máx) mediante la prueba YoYo intermittent recovery test (Bangsbo, Iaia, $\&$ Krustrup, 2008). Es una prueba de campo incremental hasta el agotamiento cuya intensidad está determinada por un audio pregrabado. Se requiere correr una distancia de $20 \mathrm{~m}$ de ida y vuelta y una recuperación activa de $10 \mathrm{~s}$ entre cada hit. Este test ha sido utilizado en población basquetbolista (Castagna et al., 2008) y considerado como un instrumento válido y confiable para determinar las respuestas fisiológicas (Krustrup et al., 2003) de los individuos. Se considera el registro del nivel y vuelta alcanzado.

\section{Agilidad}

Se aplicó el test lane agility drill (Sabin \& Marcel, 2016), el sujeto inició en una extensión de la línea de tiros libres al lado izquierdo para continuar el patrón delimitado por el área de la pintura, que en su recorrido requiere correr hacia enfrente, luego posición de defensa en avance lateral derecho, luego backpedal y finalmente defensa en avance lateral izquierdo (Kucsa \& Mačura, 2015). El recorrido se realiza de forma inversa y se anota el tiempo utilizado en realizar el recorrido. Este test se repite una segunda ocasión luego de un periodo de recuperación de cuatro minutos

\section{Velocidad 3/4 cancha}

El sujeto fue instruido a recorrer la distancia de $22.2 \mathrm{~m}$ en el menor tiempo posible desde la línea de base hasta la línea de tiros libres de la cancha 
contraria. Este test se repite una segunda ocasión luego de un periodo de recuperación de cuatro minutos

\section{Salto vertical}

Se utilizó el salto con contramovimiento CMJ (Bosco, Luhtanen, \& Komi, 1983) para valorar esta capacidad y fue necesario la colocación de una cámara de video que filmara dos ejecuciones, para cada sujeto, desde un plano coronal al lado derecho asegurando que se mantuviera el plano de ejecución visible. Cada video fue procesado por el software gratuito Kinovea v 8.15 para determinar la altura máxima alcanzada. La calibración del software requirió la filmación de un banco antropométrico con medidas de 50 x $50 \mathrm{~cm}$ para calcular cada salto (Junior, 2017)

\section{Tiros libres estáticos}

Se permitió al sujeto hasta un máximo de 10 lanzamientos a la canasta antes de iniciar con evaluación que requirió de 15 tiros desde la línea de tiros libres registrando los intentos encestados. El tiempo de cada ejecución fue auto administrado por cada participante.

\section{Tiros libres dinámicos}

Para este test, se utilizaron las medidas de la cancha de voleibol que de forma regular están marcadas en la mayoría de los gimnasios de baloncesto, tal como lo recomiendan Pojskić, Šeparović, Muratović, and Užičanin (2014) . El sujeto inicia un sprint de $18 \mathrm{~m}$ recorrido que corresponde a la longitud de la cancha de voleibol para luego recuperarse y llegar a la línea de tiros libres, recibe un pase de la pelota y ejecuta un lanzamiento. Posteriormente, se recorren de vuelta otros $18 \mathrm{~m}$ hacia la cancha contraria para ejecutar otro tiro libre. Se repite hasta completar $60 \mathrm{~s}$.

\section{Análisis estadístico}

Los datos se presentan en medias y desviación estándar. Las comparaciones estadísticas se llevaron a cabo utilizando Análisis de Varianza con PostHoc de Bonferroni con un nivel de confianza de 0.05.

\section{Resultados}

Según la Organización Mundial de la Salud (OMS) se ha establecido un IMC de 18.5-24.9 para peso normal en el que la relación estatura-peso, es considerada ideal. Valores por debajo de este rango podrían indicar desnutrición, por otro lado, valores por encima se relacionan con sobrepeso. En el caso del Grupo de Estudio (GE) se encuentran en la categoría cercana al sobrepeso, en tanto, por posición de juego se señala que los jugadores se 
encuentran desde normal hasta sobrepeso y sin diferencias significativas entre los grupos.

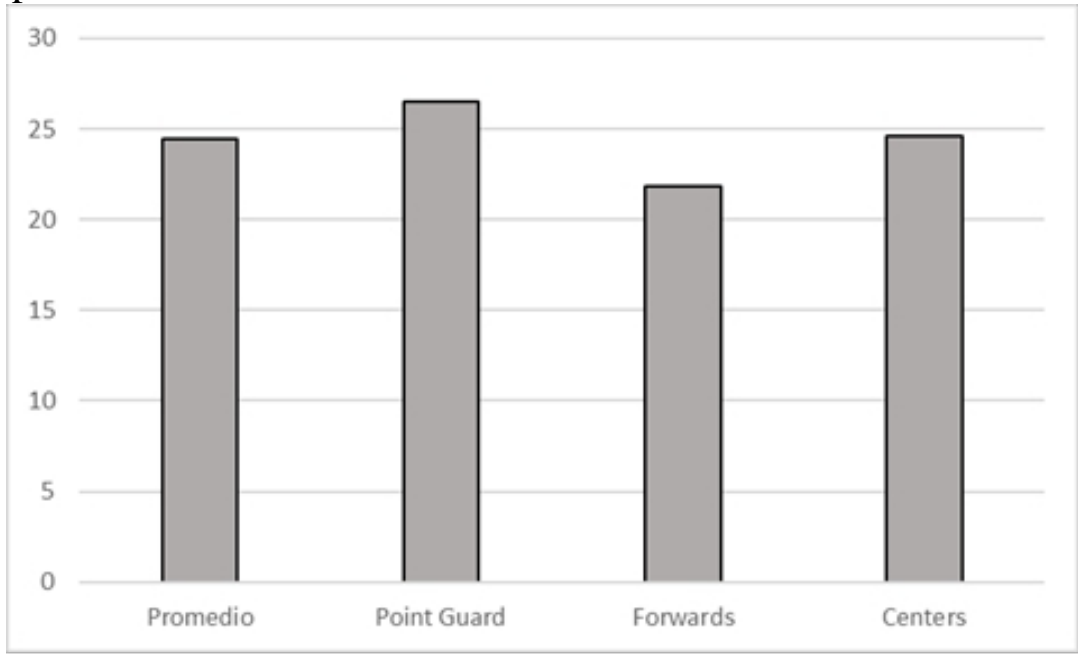

Figura 1. Representación gráfica del índice de masa corporal por cada posición de juego

En la Tabla 1 se muestran los resultados para las variables de capacidades físicas. Las comparaciones estadísticas no detectaron diferencias entre grupos para consumo de oxígeno, agilidad, velocidad salto vertical, tiros libres estáticos y dinámicos.

Tabla 1. Comparaciones en variables de rendimiento por posición de juego

\begin{tabular}{|ll|c|c|c|}
\hline & & N & Media & Desv Est \\
\hline Consumo máximo de & PG & 6 & 41.9 & 2.0 \\
oxígeno (ml·kg·min) & FG & 5 & 41.2 & 2.3 \\
& Center & 4 & 40.5 & .8 \\
& Total & 15 & 41.3 & 1.9 \\
\hline Agilidad (s) & PG & 6 & 15.9 & 0.4 \\
& FG & 5 & 15.1 & 0.7 \\
& Center & 4 & 16.6 & 1.5 \\
& Total & 15 & 15.8 & 1.0 \\
\hline Velocidad (s) & PG & 6 & 4.0 & 0.3 \\
& FG & 5 & 3.8 & 0.3 \\
& Center & 4 & 3.9 & 0.4 \\
& Total & 15 & 3.9 & 0.3 \\
\hline Salto vertical (cm) & PG & 6 & 31.5 & 4.7 \\
& FG & 3 & 34.0 & 2.3 \\
& Center & 4 & 29.5 & 2.0 \\
& Total & 13 & 31.4 & 3.8 \\
\hline TLE (\%) & PG & 6 & 59.9 & 21.9 \\
& FG & 5 & 70.6 & 18.0 \\
& Center & 4 & 48.3 & 22.0 \\
& Total & 15 & 60.4 & 21.1 \\
\hline TLD (\%) & PG & 6 & 36.1 & 28.7 \\
\hline
\end{tabular}




\begin{tabular}{|l|c|c|c|}
\hline FG & 4 & 25.0 & 16.6 \\
Center & 4 & 49.9 & 23.5 \\
Total & 14 & 36.9 & 24.6 \\
\hline
\end{tabular}

TLE $=$ Tiros Libres Estáticos; TLD= Tiros libres dinámicos

\section{Discusión}

Aunque existen métodos más precisos y novedosos para determinar composición corporal, el IMC sigue siendo una buena alternativa para diagnosticar peso saludable. Se sabe además que este parámetro puede cambiar con respecto a la edad, género, raza y edad, o por ejemplo un atleta puede tener el mismo IMC que una persona sedentaria y será posiblemente debido a mayor porcentaje de músculo que de grasa. Para el caso de los sujetos evaluados, el IMC los describe como personas con sobrepeso, y aún más, por posición de juego las jugadoras PG distan de ser acorde al rol dentro de la cancha, ya que se requiere que sean veloces y ágiles pero esto no es posible con un IMC elevado (Rexhepi \& Brestovci, 2010).

La capacidad aerobia está íntimamente relacionada con el esfuerzo continuo que una persona puede ejecutar. Valores altos de este componente fisiológico, pueden determinar el mantenimiento de periodos largos de juego reduciendo la fatiga y mejorando el rendimiento en baloncesto. Para tal efecto, los valores esperados para un equipo de baloncesto han sido reportados por Tsunawake et al. (2003) que declara un consumo de oxígeno de 56.7 $\mathrm{ml} \cdot \mathrm{kg} \cdot \mathrm{min}$ para su grupo de estudio lo que significa un $27 \%$ más de este componente fisiológico que para la muestra de este estudio. Si bien es cierto que una mayor base aerobia es esperada en deportistas, también se han encontrado valores de $46.1 \mathrm{ml} \cdot \mathrm{kg} \cdot \min$ en mujeres de ese deporte lo que sugiere la variabilidad de este componente sin distinción del nivel deportivo (Mageean, Alexander, \& Mier, 2011). Sin embargo, cabe señalar que Mageean et al. (2011) valoraron esta capacidad física en laboratorio y tapiz rodante en donde la validez ecológica se ve comprometida y por lo tanto su propia capacidad física.

Por otro lado, la agilidad es un test de uso común para selectivo de jugadores representativos de algún club o equipo deportivo, cabe señalar que en este estudio no se detectaron diferencias con respecto a la posición de juego, lo cual no es de esperarse, ya que los jugadores PG tienden a ser más veloces que el resto. Lo anterior también es señalado por Kucsa and Mačura (2015) que en su reporte declaran, además, mejores tiempos para esa prueba que nuestro grupo de estudio, también demuestra que los PG son más veloces al hacer menor tiempo en este test que los jugadores que ocupan otras posiciones de juego. De la misma forma la velocidad refleja el tiempo utilizado en recorrer $3 / 4$ de cancha, en donde se observa que los PG son los que presentan mayor tiempo, seguido de los $\mathrm{C}$ finalmente los $\mathrm{FG}$ que consistentemente son 
mejores al menos en velocidad, aunque sin diferencias estadísticamente significativas.

En lo que respecta al salto vertical, que es una cualidad necesaria en cualquier deporte y que cobra especial relevancia en el basquetbol, se observan valores relativamente bajos con respecto a otras muestras, como por lo reportan Woolstenhulme, Bailey, and Allsen (2004) quienes señalan valores de salto vertical en jugadoras de baloncesto de $49.5 \mathrm{~cm}$, mientras que Kucsa and Mačura (2015) señala que sus jugadoras poseen valores de salto por encima de los $50 \mathrm{~cm}$, por lo que se consideran áreas de oportunidad y de mejora para las jóvenes deportistas de este estudio.

Finalmente el tiro libre estático en el cual se detectaron valores promedio de $60 \%$ de efectividad, muy similares a los reportados en ligas femeniles universitarias (Harle \& Vickers, 2001) inclusive durante los registros en competencia (Zuzik, 2011). Este valor se ve fuertemente afectado cuando los sujetos se someten a un régimen de esfuerzo físico, ya que, en los tiros libres dinámicos, el porcentaje cayó hasta alrededor de $30 \%$ lo que se relaciona con la capacidad física aeróbica reducida y a la respuesta fisiológica a la fatiga.

\section{Conclusión}

Se encontraron valores homogéneos entre los grupos, lo que no es representativo de la literatura en la que PG y PF presentan mejor estado físico, al menos para ser competitivo en las ligas universitarias de México. Además de lo anterior se recomienda para futuros estudios utilizar las respuestas fisiológicas y rendimiento deportivo durante las competencias reales de baloncesto en los torneos en los que regularmente se encuentran sometidas las atletas como parte de su andar deportivo.

\section{References:}

1. Bangsbo, J., Iaia, F. M., \& Krustrup, P. (2008). The Yo-Yo intermittent recovery test. Sports medicine, 38(1), 37-51.

2. Bosco, C., Luhtanen, P., \& Komi, P. V. (1983). A simple method for measurement of mechanical power in jumping. European journal of applied physiology and occupational physiology, 50(2), 273-282.

3. Castagna, C., Impellizzeri, F. M., Rampinini, E., D’Ottavio, S., \& Manzi, V. (2008). The Yo-Yo intermittent recovery test in basketball players. Journal of Science and Medicine in Sport, 11(2), 202-208.

4. Csataljay, G., O’Donoghue, P., Hughes, M., \& Dancs, H. (2009). Performance indicators that distinguish winning and losing teams in basketball. International Journal of Performance Analysis in Sport, 9(1), 60-66. 
5. Delextrat, A., \& Cohen, D. (2009). Strength, power, speed, and agility of women basketball players according to playing position. The Journal of Strength \& Conditioning Research, 23(7), 1974-1981.

6. Harle, S. K., \& Vickers, J. N. (2001). Training quiet eye improves accuracy in the basketball free throw. The Sport Psychologist, 15(3), 289-305.

7. Junior, N. K. M. (2017). Jump teste to evaluate the volleyball player. Revista Brasileira de Prescrição e Fisiologia do Exercício (RBPFEX), 11(67), 504-508.

8. Kilic-Toprak, E., Yapici, A., Kilic-Erkek, O., Koklu, Y., Tekin, V., Alemdaroglu, U., \& Bor-Kucukatay, M. (2015). Acute effects of YoYo intermittent recovery test level 1 (Yo-YoIR1) on hemorheological parameters in female volleyball players. Clinical hemorheology and microcirculation, 60(2), 191-199.

9. Koutlianos, N., Dimitros, E., Metaxas, T., Cansiz, M., Deligiannis, A., \& Kouidi, E. (2013). Indirect estimation of VO2max in athletes by ACSM's equation: valid or not? Hippokratia, 17(2), 136.

10. Krustrup, P., Mohr, M., Amstrup, T., Rysgaard, T., Johansen, J., Steensberg, A., . . . Bangsbo, J. (2003). The yo-yo intermittent recovery test is highly reproducible, sensitive, and valid. Medicine and science in sports and exercise, 35(12), 2120-2120.

11. Kucsa, R., \& Mačura, P. (2015). Physical characteristics of female basketball players according to playing position. Acta Facultatis Educationis Physicae Universitatis Comenianae, 55(1), 46-53.

12. Latin, R. W., Berg, K., \& Baechle, T. (1994). Physical and Performance Characteristics of NCAA Division I Male Basketball Players. The Journal of Strength \& Conditioning Research, 8(4), 214218.

13. Mageean, A. L., Alexander, R. P., \& Mier, C. M. (2011). Repeated Sprint Performance in Male and Female College Athletes Matched for $\mathrm{VO}(2) \max$ Relative to Fat Free Mass. International journal of exercise science, 4(4), 229-237.

14. Metaxas, T. I., Koutlianos, N., Sendelides, T., \& Mandroukas, A. (2009). Preseason physiological profile of soccer and basketball players in different divisions. The Journal of Strength \& Conditioning Research, 23(6), 1704-1713.

15. Miller, S., \& Bartlett, R. (1996). The relationship between basketball shooting kinematics, distance and playing position. Journal of sports sciences, 14(3), 243-253.

16. Pojskić, H., Šeparović, V., Muratović, M., \& Užičanin, E. (2014). The relationship between physical fitness and shooting accuracy of 
professional basketball players. Motriz: Revista de Educação Física, 20(4), 408-417.

17. Rexhepi, A. M., \& Brestovci, B. (2010). The differences in body volume and skinfold thickness between basketball players and footballers. Int. J. Morphol., 28(4).

18. Rojas, F. J., Cepero, M., Ona, A., \& Gutierrez, M. (2000). Kinematic adjustments in the basketball jump shot against an opponent. Ergonomics, 43(10), 1651-1660. doi: 10.1080/001401300750004069

19. Sabin, S. I., \& Marcel, P. (2016). TESTING AGILITY SKILL AT A BASKETBALL TEAM (10-12 YEARS OLD). Ovidius University Annals, Series Physical Education \& Sport/Science, Movement \& Health, 16(1).

20. Tsunawake, N., Tahara, Y., Moji, K., Muraki, S., Minowa, K., \& Yukawa, K. (2003). Body composition and physical fitness of female volleyball and basketball players of the Japan inter-high school championship teams. Journal of physiological anthropology and applied human science, 22(4), 195-201.

21. Woolstenhulme, M. T., Bailey, B. K., \& Allsen, P. E. (2004). Vertical Jump, Anaerobic Power, and Shooting Accuracy Are Not Altered 6 Hours After Strength Training in Collegiate Women Basketball Players. The Journal of Strength \& Conditioning Research, 18(3), 422425.

22. Zambak, Ö. (2019). Evaluation of Maximum aerobic power, shoot speed and $20 \mathrm{~m}$. sprint powerof football players at pre-season, midseason. International Journal of Disabilities Sports and Health Sciences, 2(2), 72-77.

23. Zuzik, P. (2011). Free throw shooting effectiveness in basketball matches of men and women. Sport Science Review, 20. 